\title{
Inhibition of multiplication of herpes simplex virus by caffeic acid
}

\author{
KEIKO IKEDA $^{1,2}$, KAZUKO TSUJIMOTO ${ }^{1}$, MISAO UOZAKI ${ }^{1}$, MITSUNORI NISHIDE $^{1}$, \\ YUKIKO SUZUKI $^{2}$, A. HAJIME KOYAMA ${ }^{1}$ and HISASHI YAMASAKI ${ }^{1}$ \\ ${ }^{1}$ Division of Virology, Department of Cellular and Molecular Medicine, Graduate School of Medicine, \\ ${ }^{2}$ School of Nursing and Health Science, Wakayama Medical University, Wakayama 641-0011, Japan
}

Received March 24, 2011; Accepted May 6, 2011

DOI: $10.3892 / \mathrm{ijmm} .2011 .739$

\begin{abstract}
Hot water extracts of coffee grinds and commercial instant coffee solutions have been shown to exhibit marked antiviral and virucidal activities against herpes simplex virus type 1 (HSV-1). Specifically, it has been shown that caffeine and $\mathrm{N}$-methyl-pyridinium formate inhibit the multiplication of HSV-1 in HEp-2 cells. The present study examined the virological properties and the antiviral activity of caffeic acid against HSV-1. Caffeic acid inhibited the multiplication of HSV-1 in vitro, while chlorogenic acid, a caffeic acid ester with quinic acid, did not. These reagents did not have a direct virucidal effect. The one-step growth curve of HSV-1 showed that the addition of caffeic acid at $8 \mathrm{~h}$ post infection (h p.i.) did not significantly affect the formation of progeny viruses. An analysis of the influence of the time of caffeic acid addition, revealed that addition at an early time post infection remarkably inhibited the formation of progeny infectious virus in the infected cells, but its addition after $6 \mathrm{~h}$ p.i. (i.e., the time of the completion of viral genome replication) did not efficiently inhibit this process. These results indicate that caffeic acid inhibits HSV-1 multiplication mainly before the completion of viral DNA replication, but not thereafter. Although caffeic acid showed some cytotoxicity by prolonged incubation, the observed antiviral activity is likely not the secondary result of the cytotoxic effect of the reagent, because the inhibition of the virus multiplication was observed before appearance of the notable cytotoxicity.
\end{abstract}

\section{Introduction}

During the course of studies of the antiviral activities of various natural products and their components (1-8), we found that hot water extracts of coffee grinds and commercial instant coffee solutions exhibit marked antiviral and virucidal activities against herpes simplex virus type 1 (HSV-1), an enveloped DNA

Correspondence to: Dr Hisashi Yamasaki, Division of Virology, Department of Cellular and Molecular Medicine, Graduate School of Medicine, Wakayama Medical University, 580 Mikazura, Wakayama 641-0011, Japan

E-mail: hisay@wakayama-med.ac.jp

Key words: caffeic acid, herpes simplex virus type 1, coffee, antiviral virus (3). To identify components in the extract responsible for the observed antiviral activities of the coffee extracts, we have examined the effects of several coffee components on the multiplication of HSV-1 and have revealed that both caffeine (4) and N-methyl-pyridinium formate (7), a novel component of coffee extract, inhibit the multiplication of HSV-1 in HEp-2 cells. In addition, preliminary analyses showed that caffeic acid, which is unrelated to caffeine, has antiviral activity (6). In this report, we present the results of the virological characterization of the antiviral activity of caffeic acid against HSV-1.

Caffeic acid (3,4-dihydroxycinnamic acid) is commonly found in food mainly as chlorogenic acid (Fig. 1), an esterified form with quinic acid (Fig. 1) and chlorogenic acid is one of the most widely consumed polyphenols abundant in many plants, such as coffee, fruits, vegetables and grains. Caffeic acid has also been reported to have antioxidant activity (9).

\section{Materials and methods}

Cells and viruses. HEp-2 and Vero cells were grown in Eagle's minimum essential medium (MEM) containing 5\% fetal bovine serum (FBS). HSV-1, strain F, was used throughout the experiments. The virus was propagated in Vero cells in MEM supplemented with $0.5 \% \mathrm{FBS}$ and stored at $-80^{\circ} \mathrm{C}$ until use. The amount of virus was measured by a plaque assay as previously described (10).

Effect of the reagent on the virus yields. Caffeic acid, quinic acid and chlorogenic acid were obtained from Wako Chemicals. The reagent solution (1.0 $\mathrm{M}$ or $100 \mathrm{mM}$ ) was prepared by dissolving the reagents in hot water, and its acidity was neutralized with $1 \mathrm{~N}$ sodium hydroxide solution, followed by filtration through a Millipore DIMEX membrane (pore size $0.22 \mu \mathrm{m})$.

Monolayered cells in $35 \mathrm{~mm}$-dishes were infected with the virus at an indicated multiplicity of infection (MOI). The infected cells were further incubated at $37^{\circ} \mathrm{C}$ for the indicated period in the serum-free MEM containing $0.1 \%$ bovine serum albumin (BSA) and the indicated concentrations of the reagent. At the indicated time, the infected cells with the culture medium were harvested and the amount of total progeny virus in the culture was determined as described previously (10).

Determination of cytopathic effects and cell death. Confluent monolayers of HEp-2 cells were incubated at $37^{\circ} \mathrm{C}$ for $24 \mathrm{~h}$ in 
<smiles>O=C(O)/C=C/c1ccc(O)c(O)c1</smiles><smiles>CCOC(=O)[C@]1(O)C[C@@H](O)[C@H](O)[C@H](OC(=O)/C=C/c2ccc(O)c(O)c2)C1</smiles>

Figure 1. Structure of caffeic acid, chologenic acid and quinic acid.

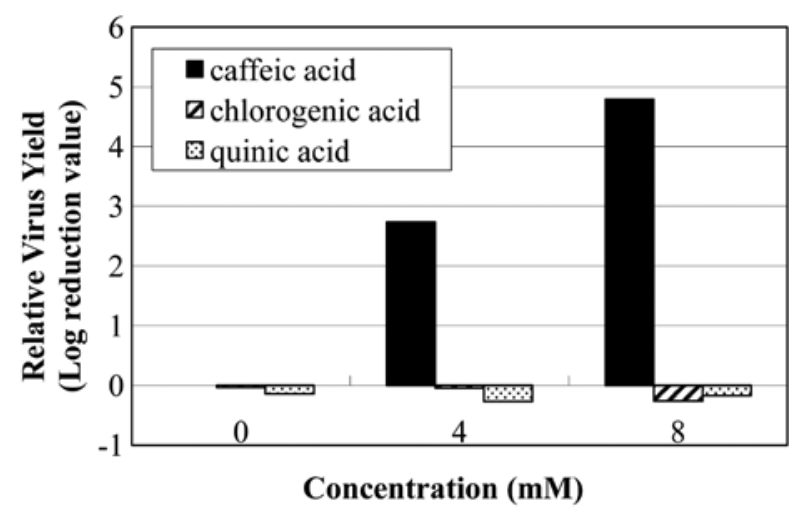

Figure 2. Effect of caffeic acid on the virus yield. Confluent monolayers of HEp-2 cells were infected with HSV-1 at an MOI of 10. The infected cells were incubated in the medium containing the indicated concentrations of caffeic acid, chlorogenic acid and quinic acid at $37^{\circ} \mathrm{C}$ for $24 \mathrm{~h}$. At the end of infection, the amounts of infectious progeny viruses were determined and were normalized to the virus yield in the absence of the reagent. Relative virus yield was calculated as a $\log$ reduction value, i.e., $\log$ [(virus yield in $\mathrm{PBS}] /[$ virus yield in the medium containing the reagent)].

serum-free MEM containing 0.1\% BSA and the indicated concentrations of the reagent. The cytopathic effects (CPE) were determined by a microscopic observation of the cells; approximate amounts of rounded cells on monolayers were estimated under a phase-contrast microscope.

To determine the extent of cell death, the monolayered cells were trypsinized to obtain single cell suspensions. After the addition of MEM containing 5\% FBS to the suspension to neutralize the trypsin and to stabilize the cells, the number of live or dead cells was determined by a dye-exclusion method with trypan blue.

\section{Results and Discussion}

Effect of caffeic acid on the multiplication of HSV-1. Caffeic acid is a naturally occurring compound and is found in plants mainly as chlorogenic acid derived by conjugation with quinic acid. The effects of these caffeic acid-related compounds on the yield of HSV-1 when the virus was propagated in the presence of various concentrations of these reagents is shown

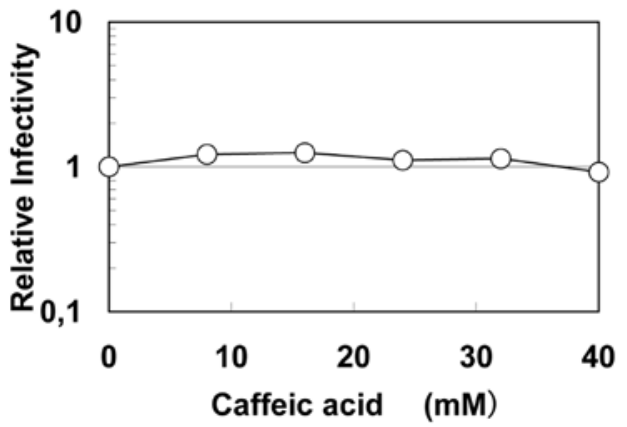

Figure 3. Effect of caffeic acid on the infectivity of HSV-1. The virus was incubated on ice for $25 \mathrm{~min}$ in PBS ( $\mathrm{pH}$ 7.4) containing the indicated concentrations of caffeic acid. After the incubation, the amounts of infectious viruses in the PBS were determined and were normalized to those in the absence of the reagent.

in Fig. 2. The virus yield at $24 \mathrm{~h}$ post infection (h p.i.) decreased remarkably with increasing concentrations of caffeic acid, while that of the other two reagents did not, in agreement with previous observations (6); at $4 \mathrm{mM}$ of the reagent, the yield of HSV-1 in the presence of caffeic acid was approximately one thousandth of that in the absence of the reagent. Under the same conditions, the presence of quinic acid or chlorogenic acid in the culture medium of the virus-infected cells did not significantly affect the virus yields. Clearer results were obtained at a higher concentration of the reagents $(8 \mathrm{mM})$. These results suggest that caffeic acid has an antiviral activity, while both chlorogenic acid and quinic acid do not. Caffeine, one of the well-known components of coffee extracts and structurally unrelated to caffeic acid, has been known to have antiviral activity against HSV-1 (4). In the present study, caffeic acid was demonstrated to have a significantly stronger (5- to 10-fold) antiviral activity than caffeine at the same concentrations.

Although chlorogenic acid does not show the antiviral activity, the molecule contains caffeic acid as its constituent. Comparing the structure of caffeic acid with that of chlorogenic acid (Fig. 1), we observed that the free carboxyl group in the propenoic acid moiety of caffeic acid is blocked in chlorogenic acid by the ester formation with quinic acid, suggesting the importance of this free carboxyl group in the antiviral activity of caffeic acid.

Effect of caffeic acid on the virus infectivity and cell viability. To examine whether the decrease in the virus-yield in the presence of caffeic acid is a result of the potential virucidal effect of the reagent, a direct effect of the reagent on the infectivity of HSV-1 was measured by incubating the virus in Dulbecco's phosphate-buffered saline (PBS) containing various concentrations of caffeic acid. The viral infectivity was not affected even by the incubation in PBS containing $40 \mathrm{mM}$ of caffeic acid (Fig. 3). This concentration is much higher than that applied for the assay for the antiviral activity described above, indicating that that there is no direct effect of caffeic acid on the infectivity of HSV-1.

The effect of caffeic acid on the viability of HEp-2 cells is shown in Fig. 4. The cells were mock-infected or infected with HSV-1, followed by incubation in serum-free medium with or without $5.0 \mathrm{mM}$ caffeic acid. HEp-2 cells do not show any cytopathic effect by the infection with HSV-1, probably due to 


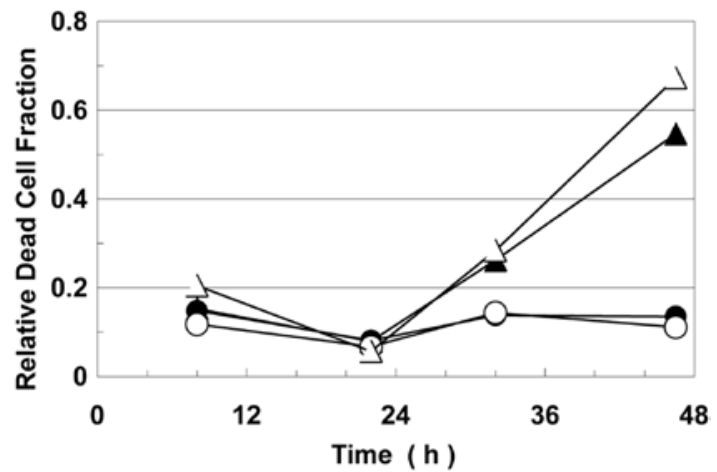

Figure 4. Effect of caffeic acid on cell viability. Confluent monolayers of HEp-2 cells were mock-infected $(O, \triangle)$ or infected with HSV-1 at an MOI of $10(\bullet, \mathbf{\Lambda})$, followed by incubation in MEM containing $0.1 \%$ BSA $(\circ, \bullet)$ or $0.1 \%$ BSA and $5.0 \mathrm{mM}$ caffeic acid $(\triangle, \mathbf{\Delta})$ at $37^{\circ} \mathrm{C}$. After incubation for $8,22,32$ and $47 \mathrm{~h}$, the cells in each culture were trypsinized to obtain a single cell suspension and the amounts of the live and dead cells were determined by a dye-exclusion test with trypan blue.

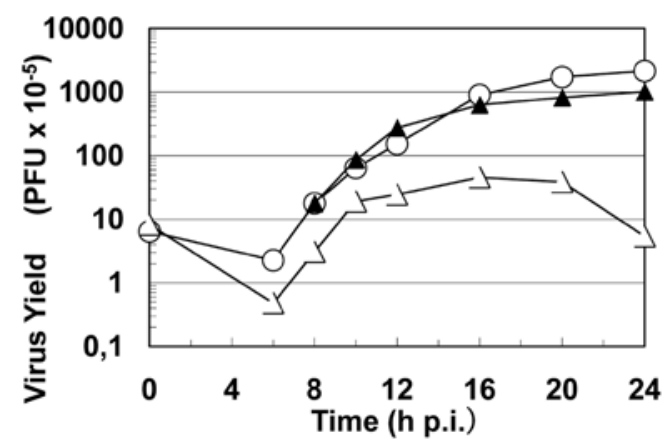

Figure 5. Effect of caffeic acid on the one-step growth curve of HSV-1. Confluent monolayers of HEp-2 cells were infected with HSV-1 at an MOI of 14. The infected cells were incubated for $24 \mathrm{~h}$ in MEM containing $0.1 \% \mathrm{BSA}$ supplemented with $(\Delta)$ or without $(0) 4.0 \mathrm{mM}$ caffeic acid at $37^{\circ} \mathrm{C}$. A group of dishes $(\mathbf{\Delta})$ received the reagent at $8 \mathrm{~h}$ p.i. At the indicated time, the amounts of total infectious progeny viruses were determined.

the presence of viral antiapoptotic gene function (11). Although the virus grew normally in the infected cells and completed its multiplication about $20 \mathrm{~h}$ p.i., both mock- and virus-infected cells (open and closed circles, respectively) did not show a significant increase in the fraction of dead cells even at $46 \mathrm{~h}$ of incubation in the absence of the reagent (Fig. 4). However, when these cells were incubated in the medium containing the reagent, the cells did not show a remarkable increase in the fraction of dead cells after $24 \mathrm{~h}$ of incubation, but thereafter, the fraction of dead cells started to increase in a time-dependent manner. Considering that the viral multiplication in the infected cells is completed at about $20 \mathrm{~h}$ p.i., these results support the notion that the cytopathic effect of caffeic acid does not result in the observed suppression of virus yield in the presence of the reagent.

One-step growth curve in the presence of caffeic acid. For the characterization of the mode of the reagent action on the multiplication of HSV-1, a one-step growth curve was examined in the presence of $4.0 \mathrm{mM}$ caffeic acid. The addition of the reagent at the beginning of virus multiplication resulted in a remarkable reduction in the number of the progeny infectious

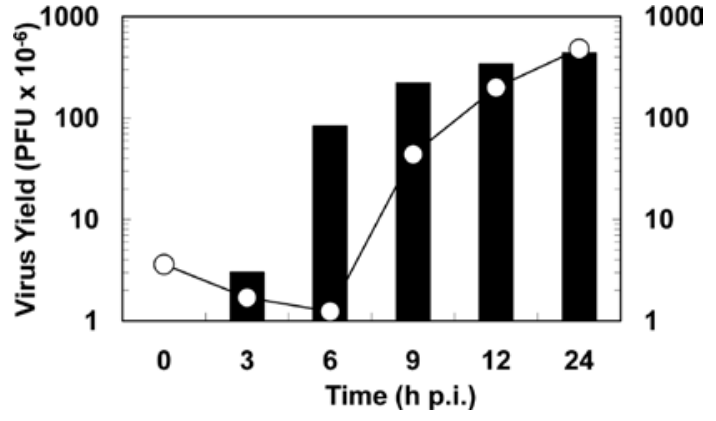

Figure 6. Effect of the time of addition of caffeic acid on the virus yield. A group of confluent monolayers of HEp-2 cells were infected with HSV-1 at an MOI of 25 and were incubated in MEM containing $0.1 \%$ BSA. At the indicated time after the infection, i) the infected culture in one dish was harvested and the amount of the infectious virus was determined to reveal a one-step growth curve (O) and ii) the infected culture in another dish received caffeic acid at the final concentration of $4.0 \mathrm{mM}$, followed by further incubation in the presence of the reagent until $24 \mathrm{~h}$ p.i. and the total progeny virus in each culture was harvested at the end of incubation to determine the amount of the final virus yield (black bars).

virus in a whole period of the virus multiplication, but the rate of progeny virus formation was not so significantly affected at the middle stage of the infection (from 6 to $10 \mathrm{~h}$ p.i.), followed by an accelerated completion of the progeny virus formation (Fig. 5). At the end of virus multiplication (at 24 h p.i.), a considerable fraction of the progeny virus disappeared from the infected cell culture, probably due to the non-specific adsorption to cells or cell debris, although we have not further examined this observation. The final yield of the progeny infectious virus produced in the presence of the reagent was less than the one hundredth of that in the absence of the reagent, in agreement with the results in Fig. 2.

In addition, when the reagent was added at $8 \mathrm{~h}$ p.i. [i.e., the time after the completion of the viral DNA replication in the HSV-1-infected cells $(12,13)]$, the formation of the progeny virus continued steadily to the end of the virus multiplication, without showing suppressive effects of the reagent, indicating that the reagent does not inhibit the virus multiplication at the late stage of the virus multiplication. These results suggest that caffeic acid inhibits the formation of progeny infectious virus in the infected cells before and/or at the step of replication of viral genome DNA in the infected cells, although an additional contribution of some other step(s) in the multiplication process cannot be excluded.

Caffeic acid-sensitive step in the HSV-1 multiplication. Previous characterization on the kinetics of the viral replication steps in HSV-1-infected cells (12) revealed that viral DNA replication occurs exclusively between 3 and $6 \mathrm{~h}$ p.i and a large amount of DNA accumulates in the infected cells when the replication of virus DNA is completed. The formation of nucleocapsids as well as the envelopment of these nucleocapsids begins at $5 \mathrm{~h}$ p.i. simultaneously with the formation of infectious progeny virus and these reactions continue in the infected cells until approximately $14 \mathrm{~h}$ p.i. Even after $14 \mathrm{~h}$ p.i., a small but significant degree of progeny virus formation continues.

To examine the target of the antiviral activity of caffeic acid in the multiplication process of HSV-1, the 'time of addition' experiment was carried out. The reagents were added to the 
infected culture at various times after the infection and the virus yield at the end of virus multiplication (black bar) was compared to the virus yield at the time of addition of the reagent (open circle) (Fig. 6). The amounts of progeny virus (black bars) were suppressed when the infected cells received the reagent at the early stage of the infection, such as at 0 or $3 \mathrm{~h}$ p.i. However, when the cells received the reagent at $6 \mathrm{~h}$ p.i., a significant but only small degree of the suppression in the amount of progeny virus was observed and the degree of the suppression became less and less with the delay of the addition of the reagent. For example, the amount of the infectious virus at $6 \mathrm{~h}$ p.i. was 1.24 (open circle at $6 \mathrm{~h}$ p.i.). When caffeic acid was added at this time point and the cell culture continued for $24 \mathrm{~h}$, the final virus yield was 83 , meaning that progeny virus formation continued. Although a virus yield of 83 was slightly less than the 440 without the addition of caffeic acid, it was much higher than the amount of the infectious virus formed at $6 \mathrm{~h}$ p.i. (1.24). Thus, it is evident that although the addition of caffeic acid slightly affects the progeny virus formation, it does not notably suppress the formation by the addition at the late stage of the infection. These results clearly show that i) the reagent interferes severely with the virus multiplication by the addition before the completion of the viral DNA replication (i.e., $6 \mathrm{~h}$ p.i.), and ii) the formation of progeny virus is affected only slightly by the addition of the reagent. These results agree with the results in Fig. 5 and the concurrence of the results in these two experiments confirms the main target of the reagent in the early stage of the virus multiplication.

In this study, we showed that caffeic acid inhibits the multiplication of HSV-1. This antiviral effect of caffeic acid is not due to a secondary result of either the direct inactivation of the virus (virucidal effect) (Fig. 2) or the cytotoxicity, but is more likely due to the specific binding of caffeic acid to the virus or molecules involved in viral replication. The results in Figs. 5 and 6 demonstrate that caffeic acid interferes with the multiplication of HSV-1 before the completion of viral DNA replication, probably at the stages of immediate early or early gene expression in the HSV-1 multiplication. Caffeic acid has been reported to have the ability to bind to certain enzymes $(14,15)$, suggesting that it may inhibit certain protein(s) necessary for virus-host interactions

\section{Acknowledgements}

The authors thank Dr Tsutomu Arakawa for stimulative discussions and assistance with the manuscript. This study is supported in part by research grants from All Japan Coffee Association.

\section{References}

1. Uozaki M, Yamasaki H, Katsuyama Y, Higuchi M, Higuchi T and Koyama AH: Antiviral effect of octyl gallate against DNA and RNA viruses. Antiviral Res 73: 85-91, 2007.

2. Yamasaki H, Uozaki M, Katsuyama Y, Utsunomiya H, Arakawa T, Higuchi M, Higuti T and Koyama AH: Antiviral effect of octyl gallate against influenza and other RNA viruses. Int J Mol Med 19: 685-688, 2007.

3. Utsunomiya H, Ichinose M, Uozaki M, Tsujimoto K, Yamasaki H and Koyama AH: Antiviral activities of coffee extracts in vitro. Food Chem Toxicol 46: 1919-1924, 2008.

4. Murayama M, Tujimoto K, Uozaki M, Katsuyama Y, Yamasaki H, Utsunomiya $\mathrm{H}$ and Koyama AH: Effect of caffeine on the multiplication of DNA and RNA viruses. Mol Med Rep 1: 251-255, 2008.

5. Furuya A, Uozaki M, Yamasaki H, Arakawa T, Arita M and Koyama AH: Antiviral effects of ascorbic acid and dehydroascorbic acids in vitro. Int J Mol Med 22: 541-545, 2008.

6. Arakawa T, Yamasaki H,Ikeda K, Ejima D, Naito T and Koyama AH: Antiviral and virucidal activities of natural products. Curr Med Chem 16: 2485-2497, 2009.

7. Tsujimoto K, Sakuma C, Uozaki M, Yamasaki H, Utsunomiya H, Oka $\mathrm{K}$ and Koyama AH: Antiviral effect of pyridinium formate, a novel component of coffee extracts. Int J Mol Med 25: 459-463, 2010.

8. Uozaki M,Ikeda K, Tujimoto K, Nishide M, Yamasaki H, Khamsri B and Koyama AH: Antiviral effects of dehydroascorbic acid. Exp Ther Med 1: 983-986, 2010.

9. Rice-Evans CA, Miller NJ and Paganga G: Structure-antioxidant activity relationships of flavonoids and phenolic acids. Free Radic Biol Med 20: 933-956, 1996.

10. Koyama AH and Uchida T: The effect of ammonium chloride on the multiplication of herpes simplex virus type 1 in Vero cells. Virus Res 13: 271-282, 1989.

11. Koyama AH and Miwa Y: Suppression of apoptotic DNA fragmentation in herpes simplex virus type 1-infected cells. J Virol 71: 2567-2571, 1997

12. Koyama AH and Uchida T: Quantitative studies on the maturation process of herpes simplex virus type 1 in Vero cells. Virus Res 10: 281-286, 1988 .

13. Roizman B and Knipe DM: Herpes simplex virus and their replication. In: Fields Virology. 4th edition. Fields BN, Knipe DM and Howley PM (eds). Lippincott-Raven, New York, pp2399-2460, 2001.

14. Staniforth V, Chiu LT and Yang NS: Caffeic acid suppresses UVB radiation-induced expression of interleukin-10 and activation of mitogen-activated protein kinases in mouse. Carcinogenesis 27: 1803-1811, 2006.

15. Kang NJ, Lee KW, Shin BJ, Jung SK, Hwang MK, Bode AM, Heo YS, Lee HJ and Dong Z: Caffeic acid, a phenolic phytochemical in coffee, directly inhibits Fyn kinase activity and UVB-induced COX-2 expression. Carcinogenesis 30: 321-330, 2009. 